\title{
Pourquoi une révision de la structure tarifaire s'impose
}

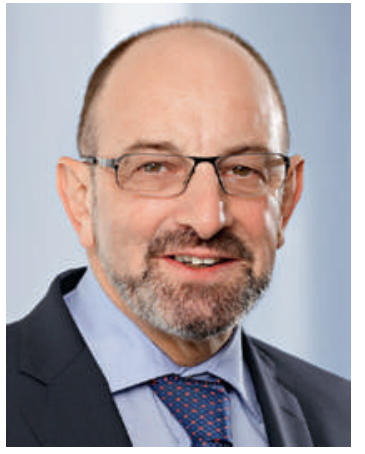

La structure tarifaire TARMED est trop complexe et privilégie certains groupes de spécialistes; elle est obsolète et ne reflète plus le quotidien actuel du cabinet médical et de l'hôpital. Tels sont les propos maintes fois entendus au sujet de la structure tarifaire TARMED qui, en tant que tarif à la prestation national, a pour tâche de reproduire correctement le travail du corps médical dans le secteur ambulatoire. Mais ces propos sont-ils exacts?

Jusqu'à présent, la preuve n'en a jamais été clairement fournie. Pourtant, le rapport du Contrôle fédéral des finances de 2010 livrait déjà des indices explicites laissant penser que certaines prestations du TARMED étaient sur- ou sous-évaluées, puisque cinq des onze exemples de cas examinés présentaient une sous-évaluation. En outre, seules quelques nouvelles positions sont venues compléter la structure tarifaire depuis son introduction en 2004 et seulement deux chapitres («Imagerie médicale» et «Traitement de la douleur») ont fait l'objet d'une révision. De ce fait, les propos cités en introduction comportent probablement une part de vérité.

\section{La structure tarifaire a pour tâche de reproduire et d'évaluer correctement les prestations dispen- sées au cabinet médical et à l'hôpital.}

Dans ce contexte, les interventions linéaires que la Confédération prévoit d'effectuer dans le cadre de sa compétence subsidiaire pour contre-financer l'amélioration de la situation des médecins de premier recours se justifient-elles? Et qu'en est-il des réductions linéaires de valeurs intrinsèques quantitatives, comme santésuisse notamment l'exige de la Confédération?

La FMH est clairement d'avis que ces interventions ne sont pas appropriées et qu'elles ne sont donc pas conformes à la loi.

La structure tarifaire n'a rien à voir avec la formation des prix, mais elle a l'obligation légale de reproduire et d'évaluer le plus correctement possible les prestations dispensées au cabinet médical et à l'hôpital. Le montant des économies découlant du rétablissement de l'adéquation ne peut être budgétisé car il dépend des résultats. Les déclarations prétentieuses de santésuisse prévoyant des économies à hauteur de 600 millions de francs sont donc complètement déplacées.

La révision de la structure tarifaire que la $\mathrm{FMH}, \mathrm{H}+$ et la CTM prévoient d'achever d'ici à fin 2015 se fonde sur un accord tripartite clairement énoncé: chacune des quelque 4500 pres- tations du TARMED doit être évaluée au moyen d'un mécanisme transparent et de principes de tarification clairs.

Ces principes et mécanismes d'actualisation de l'ensemble de la structure tarifaire seront ensuite consignés dans un manuel de tarification qui servira de base pour le développement ultérieur du TARMED.

\section{L'autonomie tarifaire doit rester la prio- rité absolue des partenaires tarifaires s'ils veulent éviter des interventions dommageables de la Confédération.}

Les objectifs que nous poursuivons sont les suivants: évaluer chaque prestation médicale de façon objective et appropriée, appliquer des règles et des mécanismes clairs, éviter un «droit du plus fort» au sein du corps médical, reproduire la réalité médicale et économique en constante mutation, simplifier la structure tarifaire et permettre sa gestion évolutive, ne pas occulter la vérité des coûts et ne pas réaliser d'économies cachées par le biais de la structure tarifaire.

Le résultat de la structure tarifaire entièrement révisée, à savoir TARMED 2.0, sera ensuite intégré dans les négociations sur les prix menées par les partenaires tarifaires qui décideront ensemble sur la base des dispositions légales combien le produit peut coûter.

Par le passé, le droit de véto exercé par les partenaires tarifaires dans le cadre de la société simple TARMED Suisse représentait le principal obstacle au développement effectif et efficace de la structure tarifaire. Celui-ci a en effet constamment bloqué la tarification de nouvelles prestations et entravé la gestion de la structure tarifaire.

En signant la convention sur la réorganisation de TARMED Suisse en juillet 2013, tous les partenaires tarifaires se sont désormais engagés, d'une part à professionnaliser le Bureau des tarifs, mais d'autre part aussi à renoncer au droit de véto afin de garantir à l'avenir l'actualisation et le développement continus de la structure tarifaire.

L'autonomie tarifaire doit rester la priorité absolue des partenaires tarifaires s'ils veulent éviter que la Confédération ne recoure à sa compétence subsidiaire en créant des déséquilibres durables dans la structure tarifaire. Le projet d'ordonnance du Conseil fédéral sur la revalorisation de la médecine de premier recours montre d'ailleurs bien l'impact que peuvent avoir ces interventions.

Dr Ernst Gähler, vice-président de la FMH, responsable du département Tarifs et conventions pour la médecine ambulatoire en Suisse 